\title{
L'asile religieux, entre lecture libérale et républicaine : quels défis pour les sociétés démocratiques?
}

\author{
Caroline Patsias et Louis Vaillancourt
}

\section{Résumé}

L'article réinscrit les analyses sur l'asile religieux au sein d'une réflexion sur la démocratie. L'asile religieux comme forme particulière de désobéissance civile ouvre à une interrogation sur la légitimité de celle-ci dans un contexte démocratique. Nous considérons d'abord les contradictions que soulève la désobéissance civile par rapport aux conceptions libérales et républicaines de l'obligation politique dans les démocraties. Nous nous penchons ensuite sur l'asile religieux, pour enfin examiner la façon dont chacune des précédentes conceptions de la démocratie peut légitimer ou, au contraire, restreindre cette forme de désobéissance civile.

\section{Abstract \\ This article resituates analyses on religious asylum in a reflection on democracy. Religious asylum as a special form of civil disobedience opens an inquiry into the legitimacy of the latter in a democratic context. We first examine the contradictions raised by civil disobedience in relation to the liberal and republican conceptions of political obliga- tion in a democracy. We then focus on religious asylum, and finally examine how each of the previous conceptions of democracy can justify or, conversely, restrict this form of civil disobedience.}

$\mathrm{L}$ e durcissement des politiques migratoires européennes et nord-américaines depuis le milieu des années 1980 jusqu'au début des années 1990 a entraîné une recrudescence de l'asile religieux, tradition qui remonterait à l'Antiquité - on songe, par exemple, au Mouvement des sans-papiers de Saint-Bernard, en France, au Sanctuary Movement, aux États-Unis, ou encore à l'asile dans l'église de la Nativité, à Bethléem, en $2002^{1}$. Pour beaucoup de sans- papiers ou de candidats à l'asile politique déboutés, l'église devient le dernier recours avant l'expulsion. Outre le report de la décision des autorités, les réfugiés espèrent une réouverture de leur dossier en gagnant, grâce à l'attention des médias, la sympathie du public. Cette pratique des églises en faveur de l'asile religieux n'a pas manqué d'offusquer les autorités étatiques, qui y voient une remise en cause de leur pouvoir et, surtout, une difficulté supplémentaire dans la gestion et la mise en œuvre des politiques publiques migratoires ${ }^{2}$. La réaction hostile de la ministre canadienne Judy Sgro face à l'asile religieux ${ }^{3}$, l'entrée des policiers, en 2004, dans l'Église unie Saint-Pierre pour procéder à l'arrestation de Mohamed Cherfi, en situation illégale au Canada par suite du refus de sa demande de statut de réfugié ${ }^{4}$, ou encore les discussions aux États-Unis sur une possible criminalisation de toute personne aidant les immigrants illégaux, témoignent sans équivoque de ce point de vue ${ }^{5}$. Ces réactions étatiques rappellent que l'asile religieux pose implicitement la question de l'obéissance civile et donc celle de son contraire « la désobéissance civile».

Originellement attribuée aux comportements de l'Américain Thoreau ${ }^{6}$, qui refusa de payer ses impôts pour protester contre les lois esclavagistes et la guerre au Mexique, la désobéissance civile demeure associée à la figure emblématique de Gandhi et à sa lutte pacifique pour l'indépendance de l'Inde. Dans l'histoire des États-Unis, elle rappelle encore les campagnes massives de désobéissance contre la guerre du Vietnam ou le mouvement pour les droits civiques. La désobéissance civile est une infraction consciente et intentionnelle, au nom de principes supérieurs. Publique, elle s'inscrit dans un mouvement collectif ${ }^{7}$. Cette définition succincte a le mérite de ne pas préciser les conditions ni les limites de la désobéissance civile. Ces dernières présupposent en effet une lecture de la démocratie et de ses engagements. Or, c'est tout l'enjeu de notre réflexion que de se pencher sur les dilemmes que posent au projet démocratique contemporain 
la désobéissance civile et l'une de ses formes particulières, l'asile religieux. Plutôt que de postuler une définition de la désobéissance civile, nous entendons souligner le lien entre conception de la désobéissance civile et vision de la démocratie. Fixer par avance les limites de la désobéissance civile serait étouffer le débat. Ouvrir celui-ci nécessite, à l'instar de Perrouty $^{8}$, de signaler que le terme " désobéissance civile » est le fruit d'une traduction malencontreuse ; " civique » eut été mieux choisi ${ }^{9}$. Le vocable « civil » ne doit pas faire oublier que si la désobéissance en question émane de la société civile, elle comporte, dans son opposition à la loi, une dimension politique déclarée. La désobéissance civile est bien une forme « moderne » - et le terme n'est pas ici sans ambiguïté ${ }^{10}$ — de résistance à l'autorité et au pouvoir.

Réfléchir sur la désobéissance civile justifie donc de revenir sur les fondements de l'obligation politique. À cet égard, le débat autour de l'asile religieux conduit à reformuler des questions récurrentes - pour ne pas dire fort anciennes - de la philosophie et de la science politique concernant les limites de l'autorité et du pouvoir politique, et le respect du droit. En reprenant une longue tradition de la réflexion politique, l'analyse qui suit entend saisir les assises théoriques du débat et ainsi mieux définir les interrogations que suscite l'asile religieux au sein de la démocratie moderne. Celle-ci induit en effet une inflexion des enjeux du débat puisque, dans les régimes démocratiques, le peuple n'est soumis qu'à sa propre loi - loi qui, de plus, dans les démocraties modernes, est fidèle aux principes des droits de la personne. Comment dès lors concevoir la légitimité de la désobéissance civile?

Un autre élément, propre à la nature même de l'asile religieux, vient infléchir cette réflexion. L'asile religieux repose sur un très ancien principe de protection des persécutés. Certains font même remonter cette tradition de sanctuaire aux directives quaurait données Dieu à Moïses, d'établir six cités refuges pour les juifs ou pour quiconque aurait tué sans intention de donner la mort ${ }^{11}$. Dans le cas de l'État moderne, c'est ce dernier qui offre l'asile aux " fugitifs » et c'est à lui que revient la tâche de déterminer qui mérite sa protection. Désormais, le statut de réfugié relève de la loi et donc de critères établis par l'État. C'est l'acquisition de ce statut qui différencie "l'étranger dans l'illégalité " ou «l'immigrant sans papiers » du réfugié au sens juridique du terme. La délivrance de ce statut revient d'ailleurs au ministère de l'Immigration, l'instance même qui déclare l'appartenance objective à un État. Ce statut juridique fluctue, selon les époques et les contextes géographiques, et de même, la sociologie des requérants varie grandement au rythme des conflits politiques. Au XIX ${ }^{\mathrm{e}}$ siècle, le candidat type à l'asile politique au Canada était le leader d'un mouvement nationaliste défait. $\mathrm{Au} \mathrm{XX}^{\mathrm{e}}$ siècle, la construction de frontières plus hermétiques ${ }^{12}$ va changer la donne et rendra le statut de réfugiés plus dépendant des normes étatiques. Le Canada a longtemps été considéré comme une terre d'asile (du moins après la fin des politiques racistes des années 1930) en raison de ses politiques migratoires qui favorisaient l'installation de refugiés sélectionnés selon les critères de l'immigration. Durant la guerre du Vietnam, nombre de soldats américains ont aussi trouvé " refuge " au Canada, sans pour autant acquérir le statut de réfugié. À partir des années 1980, l’augmentation du nombre de réfugiés a obligé le Canada à se doter d'une politique et d'instances plus spécifiques, (création du bureau des réfugiés et de l'immigration et de différentes procédures). Reprenant la Convention de Genève, est alors défini comme « réfugié » toute personne qui :

[ ... ] craignant avec raison d'être persécutée du fait de sa race, de sa religion, de sa nationalité, de son appartenance à un certain groupe social ou de ses opinions politiques, se trouve hors du pays dont elle a la nationalité et qui ne peut ou, du fait de cette crainte, ne veut se réclamer de la protection de ce pays; ou qui, si elle n’a pas de nationalité et se trouve hors du pays dans lequel elle avait sa résidence habituelle à la suite de tels événements, ne peut ou, en raison de ladite crainte, ne veut y retourner.

Depuis l'adoption de la Loi sur l'immigration et la protection des réfugiés de 2002, les demandeurs d'asile peuvent aussi être acceptés s'il est trouvé qu'ils risquent, dans leur pays, d'être torturés ou tués, ou de subir des peines ou des traitements cruels et inusités. Cependant, contrairement au projet initial, la Loi mise en vigueur exclut la possibilité d'appel. Dès le départ, des voix se font entendre pour dénoncer le manque de recours possibles et la nature politique des décisions prises par le bureau. Cette législation canadienne s'inscrit dans un contexte de restriction du droit d'asile ${ }^{13}$. À cet égard, s'il faut souligner que les demandeurs du statut de refugié ne sont pas des immigrants comme les autres - c'est du moins ce que consacre l'existence de procédures et d'un statut spécifique - les politiques relatives au droit d'asile demeurent liées au contexte des migrations et des politiques migratoires. Or ces dernières sont de plus en plus restrictives (en Europe) ou assorties de critères de sélection qui excluent les populations les plus démunies (en Amérique du Nord). À cela s'ajoutent, depuis 2001, des politiques qui tendent à privilégier la sécurité aux dépens des droits humains. Certes, ces différentes lois ne concernent pas directement la législation sur les réfugiés, mais celle-ci n'échappe pas au contexte social général par rapport aux migrants et à « l'autre ». Et force est de constater que ce contexte penche plutôt vers la criminalisation des migrants ${ }^{14}$. Les États craignent que le statut de réfugiés politiques ne soit dévoyé et qu'il ne profite à une immigration "économique " issue des pays pauvres. 
En outre, la complexification de certaines situations politiques rend plus ardu de trancher entre situation politique et situation économique, car certains pays font face à des problématiques humanitaires difficiles ou encore sont dirigés par des régimes autoritaires sans pour autant être soumis à une dictature.

Bien qu'ayant pour même fondement la protection des persécutés, les situations d'asile religieux peuvent varier selon les pays et leurs cadres normatifs. Si, au Canada, les exemples récents d'asile religieux concernaient des requérants au statut de réfugiés politiques qui s'étaient vus déboutés en Europe, et c'est notamment le cas de l'église Saint-Bernard, il s'agit essentiellement de sans-papiers. Cette différence a-telle une incidence quant à la légitimité de l'asile religieux au sein des systèmes démocratiques contemporains?

Notre réflexion comprendra trois étapes. La première partie, consacrée au cheminement de l'obligation politique, permettra, dans la deuxième partie, de montrer en quoi la modernité politique et la démocratie refondent les termes de l'obéissance politique et de cerner les enjeux que constitue, dans ce contexte, la désobéissance civile. Enfin, dans la troisième partie, nous reviendrons sur le cas particulier de l'asile religieux, en jugeant celui-ci à l'aune des précédentes théories.

\section{Obligation politique : quelques jalons théoriques}

Si la référence à une injustice (à savoir l'invocation d'un principe éthique supérieur) est au fondement des justifications de la désobéissance civile, elle est loin pour autant d'être consubstantielle à cette forme particulière de désobéissance puisqu'elle traverse le débat et les réflexions sur l'obéissance politique dès les origines. L'idée d'un droit naturel, expression d'une justice fondamentale ou divine qui peut autoriser la désobéissance à la loi des hommes i. e la loi de la cité, est déjà au cœur de l'Antigone de Sophocle. Elle teintera également les réflexions des théoriciens du Moyen-Âge sur les rapports entre Église et État, ou entre autorité divine et autorité politique ou, dans la même veine, sur la nécessité de limiter l'autorité absolue. Saint Thomas d'Aquin ${ }^{15}$ ouvre ainsi une brèche en enseignant que la loi humaine cesse d'être obligatoire en conscience lorsqu'elle contredit les commandements divins ou qu'elle opprime injustement ses sujets, car la puissance déléguée de Dieu ne saurait l'autoriser ${ }^{16}$. Les théoriciens de la souveraineté politique seront les premiers à élaborer la notion d'un pouvoir absolu et permanent d'imposer des lois aux sujets sans leur consentement et sans que ce pouvoir ne soit limité par aucune loi. Le souverain sera cependant soumis au droit divin ou naturel, qui lui interdira d'opprimer ses sujets ${ }^{17}$. Le droit divin ou naturel demeure donc la barrière à l'absolutisme du pouvoir. Il reste néanmoins que tant que la loi ne vient pas contrarier ce droit, elle doit être obéie, sans remise en question possible. La modernité, après Descartes, va introduire un déplacement de la légitimité politique de Dieu vers la Raison et l'individu en tant qu'être rationnel ${ }^{18}$. Ce déplacement ne conduit pourtant pas à un assouplissement de l'obligation d'obéissance. Comme le note Perrouty ${ }^{19}$ à la suite de Ferry ${ }^{20}$, désobéir à la loi revient à s'opposer à la raison, à la rationalité du monde. Fonder le devoir d'obéissance dans la raison ${ }^{21}$ plutôt que dans la religion n'équivaut donc pas à saper l'autorité du droit, loin s'en faut. Les théories du contrat, bien qu'offrant des différences substantielles, illustrent ce glissement vers la raison comme instance légitimatrice.

Pour Hobbes $^{22}$, le pouvoir auquel il faut obéir est absolu. C'est le besoin de protection qui explique la nécessité d'un tel pouvoir et qui est à l'origine du contrat entre les individus et l'État. Au sein de la pensée hobbesienne, l’obéissance est bien entendu dans l'intérêt de l'individu, qui échappe ainsi aux dangers de "l'état de nature ». Aussi le pouvoir protecteur est-il fatalement accompagné du pouvoir d'oppression. La liberté de l'état de nature entraîne l'insécurité et la lutte, l'assujettissement de la vie politique produit la sécurité et la paix. Les individus aliènent volontairement et irrévocablement leur souveraineté en échange de la protection de l'État. Selon Hobbes, le contrat originel est un contrat sans rétractation possible ${ }^{23}$. Certes, l'auteur du Léviathan prévoit une limite à l'obligation d'obéir si le souverain échoue à assumer sa partie du contrat - la protection des individus mais sa pensée rejette tout droit de résistance à l'oppression. L'intérêt de la protection rend caduque toute velléité d'un fondement externe, par des contenus, à l'autorité. L'ordre juridique ne repose que sur le monopole de l'État et de la force. Dans le système lockéen, au contraire, les individus ne font plus abandon de souveraineté au gouvernement. L'obéissance résulte du consentement des membres d'une communauté politique. Ces derniers s'engagent à respecter les normes juridiques établies par les autorités auxquelles ils ont volontairement confié le pouvoir. Les membres d'une communauté politique contractent de leur plein gré avec les autorités politiques, et ce contrat est révocable. Ainsi, Locke est parmi les premiers à esquisser un droit collectif de résistance à l'oppression ${ }^{24}$. Si Rousseau reste attaché à la métaphore contractuelle, il reformule le consentement à travers la notion de "volonté générale ", seule expression de la souveraineté du peuple, conçue comme inaliénable et indivisible. Le peuple, seul dépositaire de la souveraineté, n'accepte de se soumettre aux lois que parce qu'il en est l'auteur. Rousseau est donc encore loin d'évoquer la désobéissance civile au sens moderne du terme. Sa conception même de la liberté politique fait davantage référence à la liberté des Anciens qu'à celle des Modernes $^{25}$. Fait significatif, les révolutionnaires qui s'inspireront des « théories 
du contrat " affirmeront avec force l'autorité de l'État et le respect de la loi, rejoignant ainsi les théoriciens allemands, dont Kant, qui souligneront le caractère inconditionnel du devoir d'obéissance du peuple ${ }^{26}$.

\section{Désobéissance civile et démocratie}

La Modernité introduit un premier déplacement de la problématique des réflexions sur l'obéissance. Au Moyen-Âge, ces réflexions concernent les origines divines ou séculières des fondements de l'obéissance. C'est à l'aune de ces origines et de ces modalités que sont circonscrites les limites de la désobéissance. Avec la Modernité, le problème revient à justifier l'obéissance de l'homme « raisonnable » non plus selon les exigences d'une autorité extérieure et supra-humaine, mais selon celles de la raison, nouvel étalon des comportements humains. Sans conduire à un amoindrissement de la légitimité du droit, bien au contraire, celui-ci ouvre cependant la voie à «l'horizon démocratique » en s'attaquant aux fondements supra-humains de l'autorité. Aussi n'est-il pas étonnant que les théoriciens du contrat préfigurent déjà les principaux dilemmes que la désobéissance civile posera à l'État démocratique. D’abord, et comme le soulignait déjà théoriquement Hobbes, la construction de l'État moderne s'effectue à travers la conquête du monopole de la violence légitime ${ }^{27}$. Dans cette perspective, désobéir à la loi revient à saper l'autorité, voire les fondements de l'État. Ensuite, si la loi est l'expression de la volonté générale ou, pour le dire autrement, si le peuple n'est soumis qu'à sa propre loi, comment ce dernier peut-il y déroger sans revenir sur les fondements de sa propre autorité? La reconnaissance des droits opérée par la démocratie moderne va cependant conduire à une reformulation des questions précédentes. Désormais, la réflexion sur la désobéissance civile s'inscrit au sein du débat récurrent de la modernité démocratique entre défense des droits et souveraineté populaire, ou entre affirmation du juste et revendication du bien. Et la place accordée à la désobéissance civile et à ses manifestations est étroitement liée à la vision de la démocratie et aux éléments qui, en son sein, sont jugés prépondérants.

\section{Démocratie libérale et désobéissance civile}

Pour les libéraux, la souveraineté est dissociée de la loi. En d'autres termes, la loi doit relever du juste et non en priorité de l'expression de la volonté populaire. La souveraineté ne s'incarne pas dans la capacité à énoncer le droit. Il est plus important que la loi demeure l'expression de la raison et de la liberté que celle d'une volonté populaire qui pourrait être tyrannique. Cette conception explique le refus de la souveraineté comme principe hégémonique. Il s'agit, selon la vision libérale, de limiter le pouvoir, tous les pouvoirs, quelle que soit leur origine, celle-ci fût-elle populaire. Les penseurs libéraux témoignent d'ailleurs souvent d'une méfiance envers le peuple, voire "la masse ", dont on craint l'irrationalité, la violence et la menace potentielle que celle-ci représente pour l'individu et ses droits ${ }^{28}$. La liberté politique est envisagée comme un moyen de protéger la liberté et l'autonomie individuelle. C'est donc à juste titre que le libéralisme est traditionnellement associé à la liberté négative (à savoir le maximum de liberté que chacun peut avoir en vertu de la loi) et à la préservation de la sphère privée de la mainmise du pouvoir ${ }^{29}$. Cette volonté de préserver la liberté de l'individu justifie l'importance accordée à la défense des droits individuels et de la personne. Cette défense a deux conséquences, et la seconde, corollaire de la première, concerne plus directement notre propos. D'une part, l'accent est mis sur la dimension procédurale de la démocratie ${ }^{30}$, au détriment de la dimension substantielle. D'autre part, le respect des droits peut autoriser la désobéissance à la loi et le recours à des actions illégales ou violentes, au-delà de la traditionnelle opposition des libéraux aux formes plus directes de participation populaire.

Un accord sur le bien étant impossible ou difficile au sein des sociétés modernes, marquées par un polythéisme des valeurs, seul le respect des procédures " démocratiques » garantira le respect des droits individuels et rendra la vie en commun possible. Les procédures permettent donc l'exercice de la démocratie en même temps qu'elles en assurent la pérennité. La décision collective qui s'effectue à travers la règle de la majorité est le résultat de l'agrégation des préférences des acteurs, elle n'est soumise à aucune vision du bien ou, autrement dit, à aucun critère substantiel ou normatif ${ }^{31}$. La seule contrainte extérieure qui vise le résultat de la décision (substantially outcomes) a trait au respect des droits de la personne. Selon les libéraux, la règle du jeu démocratique suppose que la minorité s'inclinera devant les décisions de la majorité à une double condition : 1) l'on « rejouera » la partie, et les perdants d'aujourd'hui pourront être les gagnants de demain ; 2) la majorité victorieuse n’opprimera pas les droits fondamentaux de la minorité ${ }^{32}$.

C’est ce dernier élément, au cœur de la doctrine libérale, qui justifiera la désobéissance civile. Parce que le libéralisme impose des limites à l'autorité, même celle qui émane des gouvernements démocratiques, la désobéissance politique est légitime contre des lois démocratiques qui ne respecteraient pas les droits fondamentaux des individus. Aussi, d'un point de vue libéral, la désobéissance civile engaget-elle directement la nature et les limites de la règle de la majorité. Ceux qui pratiquent la désobéissance réclament une exception à la règle de la majorité. Ces limites et exceptions à la règle de la majorité ont un rapport direct avec les droits fondamentaux - soit avec certains principes d'équité et de traitement égal - que le libéralisme considère comme 
devant surseoir à la règle de la majorité. Les gouvernements démocratiques qui violent des droits fondamentaux outrepassent leur autorité. Lorsque «les violations sont assez graves ", les personnes dont les droits sont violés, ou d'autres qui font cause commune avec elles, peuvent légitimement résister, y compris en désobéissant à la loi ${ }^{33}$.

Le rapport aux droits non seulement justifie la désobéissance politique selon la vision libérale, mais prend naturellement un rôle de régulateur du bien - il détermine la légitimité et les limites d'une désobéissance libérale justifiée. La légitimité d'un acte de désobéissance, même passive, est alors d'autant plus fragilisée que non relative aux droits fondamentaux ${ }^{34}$. Première contrainte, forte, à la désobéissance civile, le respect des droits n'est pourtant pas la seule limite imposée par les libéraux à son sujet. Outre une réticence traditionnelle devant les manifestations collectives, les libéraux doivent en effet surmonter un défi de taille. Les droits de la personne comportent une reconnaissance de droits substantiels, mais ils sont aussi préservés par le respect d'une procédure. Dans la logique libérale, le respect de la procédure fait partie intégrante de l'équité des droits. Ainsi, si la désobéissance civile peut être un moyen pour rappeler des droits fondamentaux bafoués (et entre en ligne de compte toute l'étendue des interprétations possibles ${ }^{35}$ ), elle heurte en même temps la pierre angulaire de la pensée libérale. Il s'agit donc de déterminer « lorsque les violations sont assez graves » pour justifier la désobéissance civile ${ }^{36}$. Si les points de vue varient selon les auteurs, ces derniers demeurent cependant très prudents quant à l'espace de la désobéissance civile.

Pour Rawls ${ }^{37}$, comme pour Habermas ${ }^{38}$, la désobéissance civile ne peut être invoquée avant l'épuisement de tous les moyens légaux d'expression; elle sous-entend également l'acceptation des sanctions par le contrevenant ${ }^{39}$ et privilégie la non-violence. Rawls prône même, afin d'éviter une contestation générale qui pourrait saper les fondements du système démocratique, une coopération politique entre les minorités souffrant d'injustice ${ }^{40}$. D'autres sont cependant moins restrictifs. Dworkin rappelle que les organes de l'État sont faillibles et que, même dans les conditions de la légitimité procédurale, on ne peut exclure des injustices. Il est donc nécessaire que la « désobéissance civile soit acceptée comme composante de la culture politique d'une communauté démocratique développée " ${ }^{41}$. En autorisant le non-respect des conditions d'adoption de la norme ou des défaillances dans les procédures, la désobéissance permet d'approfondir le débat démocratique ${ }^{42}$. Il faut souligner ici que la contestation conformément aux principes libéraux porte sur la procédure et non sur la finalité de la décision.

Outre ce point, un rapide examen empirique souligne que, sitôt franchi le fossé de la théorie à la pratique, l'exercice de la désobéissance civile demeure difficile au vu des critères libéraux. La nécessité d'user, avant de recourir à la désobéissance civile, de tous les moyens légaux d'intervention est, dans bien des cas, impossible pour les acteurs sociaux. D'une part, la justice prend du temps, lequel manque souvent aux opposants. D'autre part, la situation des acteurs les empêche parfois d'avoir recours à ces moyens légaux. Au-delà de ces contraintes empiriques, une question fondamentale demeure : la désobéissance civile doit-elle être réduite à la défense des droits? À cette question, les visions républicaines de la démocratie répondent par la négative.

\section{Démocratie républicaine et désobéissance civile}

Pour les participationnistes ${ }^{43}$, réduire la démocratie à des procédures est insuffisant. S'ils reconnaissent l'aspect fondamental du respect des droits et des procédures dans la démocratie, une " démocratie forte " ${ }^{44}$ impose une participation effective des citoyens à la prise de décisions et à la gouvernance du pays - participation qui ne saurait se réduire au processus électoral et à l'établissement de préférence ${ }^{45}$. La souveraineté populaire ne s'exprime donc pas uniquement par défaut, à savoir dans le contrôle des gouvernants aux échéances électorales ${ }^{46}$, mais positivement dans l'implication au sein des procédures de décision et d'élaboration des politiques ${ }^{47}$. Les penseurs républicains voient le lien entre État et démocratie à travers la notion de souveraineté populaire, garante de l'expression du peuple et du caractère démocratique de l'État. La démocratie réclame donc, outre le respect des droits, un principe d'autodétermination. La liberté des citoyens réside dans la capacité de participer à l'élaboration de la loi ${ }^{48}$ et non pas simplement dans la possibilité d'échapper à l'emprise de l'État. La liberté civique est à la fois un moyen pour protéger la liberté individuelle ainsi que l'affirmation d'une identité, d'une appartenance à la collectivité en participant à l'édiction de la loi ${ }^{49}$. C'est la participation à la décision qui fonde la souveraineté populaire et démocratique plus que la nature de la décision elle-même.

Cette vision de la démocratie explique les traits communs à ces penseurs dans leur conception de la désobéissance civile. Cette dernière n'a plus pour seul objectif de s'opposer au non-respect des droits, elle doit aussi permettre de compenser un "déficit démocratique ou de souveraineté " ${ }^{50}$. Le terme désigne des cas où la souveraineté populaire n'a pu être engagée ou devrait être réengagée, par exemple lorsqu'une politique publique n'a pas été approuvée par les citoyens, lorsque des transformations au projet initial n'ont pas fait l'objet de consultations populaires, ou encore lorsque la situation originelle ayant présidé à la mise à l'agenda de la politique a changé. La vision de la démocratie des participationnistes, qui fonde la légitimité du régime démocratique sur le plein exercice de la souveraineté populaire, exige alors 
que celle-ci s'exprime à nouveau. Cependant, les moyens légaux mis en place pour pallier lesdits « déficits démocratiques » peuvent parfois être inefficients en raison de l'inertie des institutions ou des contraintes de temps qu'impose une réforme du système ${ }^{51}$. Il est par ailleurs impossible, pour tout système politique, d'anticiper la totalité des déficits qu'il génère et de prévoir les moyens légaux pour y remédier. Dans cette perspective, la désobéissance civile s'avère une façon d'éviter l'amputation de la souveraineté du citoyen à cause de contraintes systémiques.

À l'opposé de la désobéissance selon les libéraux, qui vise en priorité un changement ou une remise en cause dans la nature de la politique au nom du respect des droits, l'objet premier de la désobéissance civile demeure le réengagement de la souveraineté ${ }^{52}$. Ainsi la vision républicaine élargit-elle le spectre des options politiques et celui de l'usage de la désobéissance civile : celle-ci est directement reliée à l'exercice de la souveraineté et n'est pas perçue comme un moyen de dernier ressort. Cependant, du même coup, la désobéissance civile se trouve intrinsèquement limité $e^{53}$. Si son but premier est de restaurer un déficit démocratique, elle n'a pas pour objectif de répondre à des considérations concernant les résultats de la politique elle-même. À cette limite téléologique s'en ajoutent d'autres. Si la désobéissance civile contribue à un élargissement de la démocratie en permettant le prolongement de la souveraineté, elle constitue également une menace pour cette dernière. En remettant en question les lois du peuple, la désobéissance civile n'affaiblitelle pas la souveraineté populaire qui l'a initiée? Un excès de désobéissance civile, en colonisant la sphère entière de l'activité politique, pourrait évincer toute autre forme légale d'action politique et minerait ainsi l'approfondissement de la démocratie que la désobéissance civile est censée défendre. Paradoxalement, les républicains se trouvent devant un écueil similaire à celui que rencontrent les libéraux : comment juger du déficit démocratique qui autorisera le recours à la désobéissance civile? Ces fondements théoriques, qui déterminent les cadres de politiques concrètes, permettent de mieux saisir les questions soulevées par l'asile religieux dans les démocraties modernes.

\section{L'asile religieux : une double lecture, libérale et républicaine}

L'asile religieux peut faire l'objet d'une double lecture, libérale et républicaine. Une telle lecture à la fois autorise une meilleure appréhension des dilemmes que ce type de désobéissance pose aux démocraties contemporaines et souligne dans quelle mesure ces deux courants de pensée ont encadré cette forme de contention. L'analyse impose cependant de distinguer entre les cas de demandeurs d'asile politique et ceux qui relèvent d'une contestation des politiques migratoires, les arguments pouvant sensiblement varier selon les situations.

\section{Une lecture libérale de l'asile religieux}

À bien des égards, la lecture libérale fait écho aux arguments soulevés par les acteurs. D’abord, la législation canadienne, qui ne permet pas le recours en appel des requérants à l'asile politique déboutés ${ }^{54}$, heurte la philosophie libérale au sein de laquelle la procédure d'appel constitue une garantie supplémentaire des droits des individus. Le cas est d'autant plus problématique qu'au Canada, la décision est désormais rendue par un juge plutôt que deux, ce qui implique que le dossier de tout candidat n'est soumis qu'à un seul point de vue. Le sort d'un demandeur du statut de réfugié au Canada est donc décidé par une unique personne, sans aucune possibilité d'appel sur le fond. Or, comme le faisait remarquer le Conseil canadien pour les réfugiés en $2004^{55}$, un Canadien qui conteste une simple contravention de stationnement a droit à l'appel, alors que le demandeur du statut de réfugié, dont la vie peut être en danger, en est privé. Ensuite, et toujours du point de vue du respect des droits, les défenseurs de l'asile religieux peuvent pointer que, outre l'examen de la situation du demandeur, les législations (notamment européenne et canadienne) invoquent le " droit à une vie normale » ou encore le " meilleur intérêt de l'enfant » et que, conséquemment, le verdict des autorités peut ne pas avoir assez tenu compte des liens que le réfugié a établi dans le pays d'accueil. Enfin, la crainte des libéraux qui voient dans la désobéissance civile une mesure de recours in extremis ne peut être opposée aux pratiques des congrégations, et particulièrement à celles de l'Église unie du Canada ${ }^{56}$ qui insiste lourdement sur le caractère exceptionnel du recours.

Cependant, l'acceptation des sanctions qu'impose la définition libérale de la désobéissance civile est également problématique, du moins pour les personnes bénéficiant de l'asile ${ }^{57}$. Pour les demandeurs d'asile éconduits, accepter la sanction équivaut à se plier au retour dans leur pays d'origine, et c'est justement pour échapper à ce retour qu'on fera appel, en dernier ressort, à la désobéissance civile. Certes, le réfugié au sein du sanctuaire ne cache pas ses intentions aux autorités étatiques (le fondement de la désobéissance civile étant d'ailleurs la publicité de l'action entreprise) et rien n'empêche légalement celles-ci de briser la tradition de sanctuaire en pénétrant dans l'enceinte religieuse. Néanmoins, selon nous, cet argument n'est pas suffisant pour invalider totalement la précédente problématique. À travers l'asile, il y a bien tentative d'échapper à la sanction. Dans le cas particulier de l'asile religieux, du moins pour celui qui demande la protection du sanctuaire, les fins de la désobéissance civile (pour souligner l'injustice de la déportation et y échapper) sont confondues avec la sanction que l'État exercera sur le 
réfugié si le ministre de l'Immigration, qui dispose d'un droit d'intervention, n'est pas convaincu par les arguments de l'intéressé. Il faut cependant remarquer que ce constat ne concerne pas uniquement l'asile religieux, mais aussi d'autres cas de désobéissance civile. De ce point de vue, l'asile religieux ne fait qu'illustrer certaines des ambiguités de la pensée libérale à l'endroit de la désobéissance civile.

La protection du sanctuaire envers des immigrants irréguliers est plus problématique pour la pensée libérale. Leurs défenseurs peuvent s'appuyer sur l'idée que l'étranger a les mêmes droits fondamentaux que le citoyen (hormis ceux de voter, d'être élu, de recevoir une éducation dans la langue de la minorité, et d'entrer et de rester sur le territoire canadien). Entre donc en jeu la concurrence entre les principes et l'interprétation des droits fondamentaux ou des situations politiques des pays d'où proviennent ceux qui réclament la protection du sanctuaire. En France, le mouvement d'appui aux sans-papiers de l'église Saint-Bernard conteste la teneur des "lois Pasqua ", qui restreignent l'accès à la nationalité française et privent de nombreux étrangers résidant en France du droit de séjour. Ce mouvement s'appuie sur de grands principes humanitaires qui, sans se référer explicitement à ceux du droit international, engagent tout de même une vision large des droits de la personne et des libertés individuelles. Les défenseurs de la légitimité de l'action des réfugiés dans l'église dénoncent en outre une législation qui, de plus en plus contraignante, plonge dans l'illégalité des gens dont le statut juridique était jusqu'alors conforme à la loi. L'irrégularité de certains immigrants ne serait donc pas le fait de ceux-ci, mais plutôt attribuable à des modifications à la loi postérieures à une venue légale, modifications qui rendent le renouvellement de leur statut impossible.

Un dernier élément fragilise la légitimité de l'asile religieux des sans-papiers aux yeux des libéraux. Ces derniers ont une longue tradition de méfiance à l'endroit du désordre, et certains éléments de la protection offerte aux sanspapiers par les sanctuaires peuvent s'y rattacher, notamment la dimension collective plutôt qu'individuelle de l'action.

\section{Une lecture républicaine de l'asile religieux}

Pour les républicains, la distinction entre demandeurs d'asile et sans-papiers se révèle moins pertinente. Selon la perspective républicaine, il relève de la souveraineté de chaque État de se prononcer sur les critères de la citoyenneté et de l'appartenance nationale. La capacité à faire respecter les frontières géographiques et symboliques est un élément constitutif de la souveraineté étatique et populaire ${ }^{58}$. Cet argument peut justifier l'exceptionnalité de l'absence de recours au sein de la procédure. Au paradigme récent qui rend possible la désobéissance civile en raison d'un défaut dirimant, la vision républicaine oppose le paradigme traditionnel de la souveraineté territoriale.

Ce que ne peut soutenir en revanche la conception républicaine, c'est la non mise en vigueur d'un principe de recours inscrit dans la loi, comme c'est le cas au Canada. La non-application des lois existantes qui sont, selon la perspective républicaine, l'expression de la souveraineté du peuple non seulement est de nature non démocratique, mais elle s'oppose à la souveraineté.

Au Canada, l'Église accorde l'asile religieux si elle est convaincue que la personne risque la torture ou d'autres formes de persécution dans son pays d'origine et que ses droits fondamentaux n'ont pas été respectés. Or, dans une perspective républicaine, seul l'État peut se prononcer sur la question. Au Moyen-Âge, la pratique du sanctuaire correspondait à l'affirmation d'un ordre concurrentiel à celui de l'État et du droit civil. Si la modernité a consacré la victoire du séculier au sein des démocraties occidentales, la pratique a cependant perduré. L'asile religieux contemporain relève directement de cette mission et de ses fondements symboliques. Le terme même de sanctuaire souligne la dimension spirituelle de cette mission, qui est d'ailleurs clairement exprimée dans les discours des autorités ecclésiastiques qui invoquent une tradition universelle fondée sur la compassion, l'hospitalité, la solidarité et l'amour fraternel ${ }^{59}$. Selon les partisans de la république, l’Église vient ici empiéter sur des prérogatives qui ne sont pas de son ressort.

La vision républicaine n'est pas dénuée d'ambiguïté. Si sa conception de l'État et de la supériorité de la volonté populaire qu'il incarne a tendance à restreindre l'asile religieux, la vision participationniste qui met l'accent sur l'implication politique des citoyens en dehors des processus électoraux peut favoriser une définition plus large de la citoyenneté. Ainsi, aussi bien les sans-papiers que les réfugiés réclamant l'asile politique peuvent être économiquement et sociologiquement citoyens même s'ils demeurent juridiquement et politiquement des étrangers. La doctrine républicaine, ou du moins, la vision participationniste, peut être réceptive à cette conception de la citoyenneté. De même, la légitimité de la désobéissance civile des citoyens et des membres du sanctuaire qui vont rendre possible l'asile religieux peut profiter d'une perspective participationniste qui est traditionnellement en faveur de la participation et de l'action collective.

Cette dernière remarque rappelle que l'asile religieux en tant que forme particulière de désobéissance civile s'inscrit au sein de la problématique de l'action collective et de la façon dont la contestation et la résistance sont appréhendées au sein des sociétés. Un premier argument serait de signaler l'opposition entre action collective violente et non violente. Cette opposition est cependant moins évidente qu'il n'y paraît. La violence est aussi une notion subjective 
reliée aux mœurs sociales et politiques d'une époque. Le droit, à certains égards, ne fait que sanctionner un état des mœurs (voire des rapports de force) qui a été influencé par les résistances citoyennes précédentes. Par exemple, le fait que la grève soit reconnue comme un droit fut l'objet de luttes et de nombreuses actions collectives. Si l'on revient à la désobéissance civile, celle-ci a grandement contribué à l'élargissement des droits de minorités, des opprimés. Comme le souligne Marcuse ${ }^{60}$, la résistance, l'opposition sont toujours une violence faite à «l'ordre établi », à « la violence institutionnalisée » qui est celle de l'État, jusqu'à ce que l'opposition remporte la bataille de la légitimité. Dans une telle perspective, le recours à la violence " effective " ne peut être qu'une question de tactique. En renonçant à la violence, la désobéissance civile entend ne pas répondre à la force par la force ; cette stratégie peut aussi, surtout à l'ère contemporaine de la médiatisation, favoriser la sympathie du public. Elle souhaite conquérir l'intérêt général à travers la « justesse " de la cause défendue, cette dernière dépassant le cadre de la loi positive. La désobéissance civile et l'asile religieux puisent leurs racines dans une ancienne notion qu'il existe un droit supérieur ou une loi supérieure à la loi positive. Cette conception a été fondamentale au développement des libertés des individus et, plus généralement, à l'avancée de la démocratie. Dans le contexte contemporain de criminalisation de l'action collective ${ }^{61}$, elle demeure un recours plus que jamais nécessaire.

\section{Notes}

1. Pour le mouvement des sans-papiers de l'église SaintBernard en 1996, se reporter notamment à louvrage de T. Blin, Les sans-papiers de léglise Saint-Bernard. Mouvement social et action organisée (Paris : L'Harmattan, 2006). Le "Sanctuary Movement » aux États-Unis était un mouvement politique et religieux d'environ 500 congrégations qui ont aidé des réfugiés en provenance d'Amérique centrale en les mettant à l'abri du service de l'immigration et de la naturalisation. Le mouvement s'est épanoui entre 1982 et 1992. Pour plus de détails, voir le site du mouvement $:<$ http:// www.newsanctuarymovement.org/movement.html> (page consultée le 2 novembre 2009). Quant à la protection accordée par l'église de Bethléem, voir le site infocatho : $<\mathrm{http} / / /$ infocatho.cef.fr/fichiers_html/archives/deuxmilledeux/ semaine14/22nx14mod.html>.

2. Difficulté d'autant plus grande que ces politiques sont, depuis les événements du 11 septembre, plus étroitement reliées aux enjeux sécuritaires et à la surveillance des frontières.

3. Voir son entrevue à la Canadian Press du 4 juillet 2004.

4. Voir la page de Radio-Canada : <http://www.radio-canada. ca/regions/Quebec/nouvelles/200403/05/006-cherfi_ arrestation.shtml>.
5. Voir le Sensenbrenner-King Bill de 2006. Le but premier du projet de loi était de criminaliser les sans-papiers ; il comportait également des dispositions visant les personnes et les groupes offrant une aide humanitaire aux migrants irréguliers.

6. H. D. Thoreau, On the Duty of Civil Disobedience (New York: Holt, Rinehart and Winston, 1948).

7. H. A. Bedau, "On Civil Disobedience," Journal of Philosophy 58, no 21 (1961): 653-661. Voir également J. Mellon et J. Sémelin, La non-violence, (Paris : PUF, 1994)

8. P-A. Perrouty, éd., Obéir et désobéir : Le citoyen face à la loi (Bruxelles : Éditions de l'Université de Bruxelles, 2000): 7.

9. J. Sémelin revient de façon très précise sur cette distinction dans un article de l'Express du 27/09/97 : <http://www. lexpress.fr/informations/la-desobeissance-civique-estelle-legitime_621117.html> (page consultée le 2 novembre 2009).

10. C'est ici le terme, étroitement relié aux revendications et aux écrits de Thoreau, plus que le comportement qui est moderne. L'idée du refus d’obéir à la loi est un thème récurrent de la science politique et un tel comportement ne saurait, en outre, être réduit à la modernité ; il est au cœur de la tension dramatique antique. Il est cependant vrai que la question de la désobéissance se pose différemment dans un système démocratique moderne, c'est d'ailleurs tout l'enjeu de la présente réflexion.

11. C. Stasny et G. Tyrnauer, Sanctuary for Refugees? A Guide for Congregations (Toronto : The United Church of Canada, 2004).

12. Ainsi Stephen Zweig, dans Le monde d'hier - Souvenirs d'un Européen, évoque le temps où des passeports nétaient pas nécessaires pour voyager.

13. Conseil des réfugiés, 2004. Voir la page : <http://www. ccrweb.ca/keyissuesfr.htm>.

14. Voir la note 5.

15. Thomas d'Aquin, Somme théologique, Ia-IIae, Q96. art. 4.

16. P-A. Perrouty, « Légitimité du droit et désobéissance » dans P-A. Perrouty, éd., Obéir et désobéir : Le citoyen face à la loi, Bruxelles, Éditions de l'université de Bruxelles (2000) : 62.

17. Q. Skinner, Les fondements de la pensée politique moderne (Paris : Albin Michel, 2005).

18. B. Frydman, Les transformations $d u$ droit moderne (Bruxelles: Story-Scientia, 1999).

19. Perrouty, supra note 7: 59-78.

20. J-M. Ferry, Philosophie de la communication. Justice politique et démocratie procédurale (Paris : Éditions du Cerf, 1994).

21. A. M. Bickel, The Morality of Consent (New Haven : Yale University Press, 1975).

22. T. Hobbes, Léviathan (Paris : Éditions Sirey, 1971).

23. H. Höffding, Histoire de la philosophie moderne, Tome I (Paris : Éditions Félix Alcan, 1906).

24. P. Gérard, Droit et démocratie. Réflexions sur la légitimité du droit dans la société démocratique contemporaine (Bruxelles: Éditions De l'Université de Bruxelles, 1995); P. Gérard, "Les 
justifications de l'autorité du droit dans la société démocratique et la désobéissance civile," in Perrouty, supra note 7: 79-93.

25. J-F Spitz, La liberté politique : essai de généalogie conceptuelle (Paris : PUF, 1995)

26. P-A. Perrouty, éd., Obéir et désobéir: Le citoyen face à la loi (Bruxelles : Éditions de l'Université de Bruxelles, 2000).

27. M. Weber, Économie et Société (Paris : Plon, 1971); T. Skocpol, States and Social Revolutions : A Comparative Analysis of France, Russia, and China (Cambridge : Cambridge University Press, 1979). La fin du Moyen-âge correspond à lémergence de l'individu qui, sémancipant de sa communauté originelle, va contracter avec l'État au moyen du pacte sécuritaire. Voir B. Badie et G. Hermet, Politique comparée (Paris : Colin, 2001).

28. Y. Papadopoulos, Démocratie directe (Paris : Economica, 1998).

29. Ceci explique, comme le remarque pertinemment Justine Lacroix, que les libéraux tentent davantage de répondre à la question "Jusqu'où le gouvernement s'ingère-t-il dans mes affaires » plutôt qu’à «Qui gouverne? » Voir J. Lacroix, Communautarisme versus libéralisme : quel modèle d'intégration politique? (Bruxelles : Éditions de l'Université libre de Bruxelles, 2003).

30. B. Manin, «L'idée de démocratie délibérative dans la science politique contemporaine. Introduction, généalogie et éléments critiques. Entretien avec Bernard Manin ", Politix 15, no 57 (2000) : 37-55. Évidemment, sont soulignés ici les grandes lignes de la pensée libérale qui peuvent aussi conduire à gommer quelques subtilités de cette pensée d'autant quelle est riche de plusieurs courants et de maints auteurs. Les libéraux n’ont pas complètement écartés la dimension substantielle, comme les participationnistes et les républicains se soucient également de la dimension procédurale. Il s'agit ici de souligner de nouveau les points les plus significatifs de ces courants de pensée.

31. P. Jones, "Political Equality and Majority Rule," The Nature of Political Theory (Oxford: Clarendon Press, 1983) : 155182.

32. B. Manin, Principes du gouvernement représentatif (Paris : Calman-Lévy, 1995); G. Sartori, Théorie de la démocratie (Paris : Armand Colin, 1973).

33. J. Rawls, Théorie de la justice (Paris : Éditions Du seuil, 1987); J. Habermas, Le droit et la force. Écrits politiques (Paris: Cerf, 1990); R. Dworkin, Une question de principe (Paris : PUF, 1996).

34. R. Dworkin, Une question de principe (Paris : PUF 1996).

35. Le respect des droits lui-même peut être interprété de diverses façons, et plusieurs droits peuvent entrer en concurrence.

36. Idéalement, selon l'esprit libéral, désobéissance civile et processus juridique servent tous deux la défense des droits et devraient donc opérer de concert.

37. J. Rawls, supra note 23.
38. J. Habermas, Le droit et la force. Écrits politiques (Paris : Cerf, 1990).

39. Cette dimension est importante pour les libéraux, car elle exprime la fidélité à la loi (et donc aux fondements du système démocratique) et prouve que lacte est en réalité " politiquement responsable et sincère » et qu'il est conçu pour toucher le sens de la justice du public. Voir J. Rawls, supra note 23.

40. J. Habermas, Débats sur la justice (Paris : Cerf, 1997).

41. R. Dworkin, Une question de principe (Paris : PUF, 1996): 133 et suivantes.

42. J. Habermas, supra note 30 ;

43. C'est certes prendre quelques libertés que d'associer républicains et participationnistes, néanmoins les positions sur la désobéissance civile se recoupant, nous avons choisi de réunir les deux courants de pensée.

44. B. Barber, Strong Democracy: Participatory Politics for a New Age (University of California Press, 1984).

45. C. Mouffe, The Democratic Paradox (Londres/New York : Verso, 2000).

46. Voir, entre autres, J. Schumpeter, Capitalisme, socialisme et démocratie (Paris : Petite bibliothèque Payot, 1946). Cette vision de la "préférence » de la démocratie nourrira les tenants du Public Choice. Voir J. Bohman et W. Rehg, Deliberative Democracy: Essays on Reason and Politics (Cambridge : MIT Press, 1997).

47. Cette participation à l'élaboration des lois implique d'ailleurs pour certains la reconnaissance de l'activisme, laquelle favorise un approfondissement de la démocratie. Voir G. Baiocchi, "Participation, Activism and Politics," in A. Fung et E. Wright (dir.), Deepening Democracy (Londres : Verso, 2002).

48. On reconnaît ici l'héritage rousseauiste : l'homme est né libre et partout il est dans les fers. La liberté politique gît dans la capacité des citoyens de choisir leurs propres fers. "L'obéissance à la loi quon s'est prescrite est la liberté. » (1762, Livre I, Chapitre 8).

49. H. Arendt, "La désobéissance civile », in Du mensonge à la violence (Paris : Pocket, 1994); R. Putnam, Bowling alone: The collapse and Revival of American Community (New York : Simon and Schusters, 2000).

50. D. Markovits, "Democratic Disobedience," The Yale Law Journal 114, no 8 (2005): 1897-1952.

51. P. Singer, Democracy and Disobedience (Oxford: Clarendon Press, 1973).

52. D. Markovits, supra note 40.

53. La désobéissance civile concerne le fait du réengagement démocratique et non les résultats ou les fins de celui-ci.

54. Notre propos concerne ici surtout l'exemple canadien.

55. Voir note 13.

56. C. Stasny et G. Tyrnauer, Sanctuary for Refugees? A Guide for Congregations (Toronto : The United Church of Canada, 2004).

57. Les églises, comme ceux qui soutiennent celui y ayant trouvé refuge, acceptent sans ambiguïtés le risque de 
sanction et, fidèles encore aux préceptes libéraux, insistent sur le caractère pacifique des moyens d'action utilisés. Voir : L. Mackenzie Shepherd, "Foi et désobéissance civile," Vivre Ensemble 12, no 41 (2004): 4-6.

58. M. Walzer, Sphères de justice. Une défense du pluralisme et de l'égalité (Paris : Seuil, 1997).

59. A. Jacob, "La violation d'un sanctuaire est un geste répréhensible," Vivre Ensemble 12, no 41 (2004) : 7-9.

60. H. Marcuse, "The problem of Violence and the radical opposition." Five Lectures. (Boston : Beacon, 1970). Le texte se trouve aussi à la page : <http://www.marcuse.org/ herbert/pubs/60spubs/67endutopia/67EndUtopiaProbVio 1.htm> (consultée le 3 novembre 2009).
61. D. Della Porta et O. Filieule, Police et manifestants, Maintien de l'ordre et gestion des conflits (Paris: Presses de Sc. Po, 2006).

Caroline Patsias est professeure au département de science politique de l'Université du Québec à Montréal (UQAM). Elle s'intéresse plus particulièrement aux transformations de la démocratie et de la citoyenneté.

Louis Vaillancourt est étudiant à la maîtrise en politique appliquée, à l'Université de Sherbrooke. Ses réflexions portent sur les pratiques du multiculturalisme au sein des démocraties modernes. 